\title{
Reversible posterior leukoencephalopathy syndrome: single photon emission computerized tomography observations
}

\author{
Salvadeeswaran Meenakshi-Sundaram¹, Sathyam Senthilnathan², Kaliappan Gurusamy Srinivasan ${ }^{3}$, \\ Somalinga Nagendran Karthik ${ }^{1}$, Pandi Suresh ${ }^{1}$, Somasundaram Palanirajan ${ }^{4}$ \\ ${ }^{1}$ Department of Neurosciences, Apollo Speciality Hospitals, Madurai 625020, Tamil Nadu, India. \\ ${ }^{2}$ Department of Nuclear Medicine, Meenakshi Mission Hospital and Research Centre, Madurai 625020, Tamil Nadu, India. \\ ${ }^{3}$ KGS Advanced MR and CT Scan Centre, Madurai 625020, Tamil Nadu, India. \\ ${ }^{4}$ Department of Nephrology, Apollo Speciality Hospitals, Madurai 625020, Tamil Nadu, India.
}

Correspondence to: Dr. Salvadeeswaran Meenakshi-Sundaram, Department of Neurosciences, Apollo Speciality Hospitals, Lake View Road, KK Nagar, Madurai 625020, Tamil Nadu, India. E-mail: drsundarsms@yahoo.com

How to cite this article: Meenakshi-Sundaram S, Senthilnathan S, Srinivasan KG, Karthik SN, Suresh P, Palanirajan S. Reversible posterior leukoencephalopathy syndrome: single photon emission computerized tomography observations. Neuroimmunol Neuroinflammation 2017;4:28-32.

Article history:

Received: 04-03-2016

Accepted: 22-12-2016

Published: 20-02-2017

Key words:

Reversible posterior

leukoencephalopathy,

single photon emission

computerized tomography

imaging,

accelerated hypertension,

crossed cerebellar diaschisis

\section{ABSTRACT}

The authors report clinical correlations of single photon emission computerized tomography (SPECT) findings in reversible posterior leukoencephalopathy (RPL). These are observations that have not received wide attention in literature. A 31-year-old hypertensive gentleman, on discontinuing antihypertensive medications, presented with vomiting, headache, focal motor to bilateral tonic-clonic seizures, altered sensorium, right gaze palsy and right hemiparesis. Accelerated hypertension was noted and he improved well with antihypertensive and anticonvulsant therapy. While cranial magnetic resonance imaging (MRI) revealed extensive bilateral lesions, SPECT imaging revealed perfusion defects involving bilateral basal ganglia, left parieto-occipital, right cerebellar and right occipital regions, which corresponded with clinical deficits on examination. While MRI is the standard of care for the evaluation of RPL, this case suggests that SPECT abnormalities may be better localized to the pathogenic lesions. Furthermore, this may begin to explain the pathophysiology of injury in RPL.

\section{INTRODUCTION}

The syndrome of reversible posterior leukoencephalopathy (RPL) was first described by Hinchey et al. ${ }^{[1]}$ who reported the clinical and radiological features in 15 patients. The clinical findings included headache, vomiting, confusion, seizures, cortical blindness and other visual abnormalities. ${ }^{[1]}$ Computerized tomography (CT) and magnetic resonance imaging (MRI) studies in these patients revealed extensive bilateral white matter abnormalities suggestive of subcortical and cortical edema without

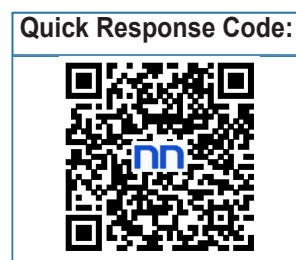


infarction especially in the posterior regions of the cerebral hemispheres. ${ }^{[2]}$ While such imaging features have now been well documented, data describing the functional imaging characteristics in this syndrome are rare..$^{[3-6]}$ The pathophysiology of RPL syndrome involves cerebral autoregulatory dysfunction. Acute rise of blood pressure beyond the upper limit of cerebral autoregulation leads to dilatation of arterioles dilate resulting in cerebral hyperperfusion in a pressurepassive manner. This leads to breakdown of the blood brain barrier resulting in extravasation of fluid and blood products into the brain parenchyma. ${ }^{[7]}$ Early changes in neurological diseases can be identified by imaging regional blood flow which is possible using single photon emission computerized tomography (SPECT) imaging which is sensitive to local metabolism and has been utilized in conditions like dementias, epilepsy and traumatic brain injury. ${ }^{[8]}$ SPECT studies may reveal focal areas of hypoperfusion that are discordant with findings of MRI or CT. For instance, in traumatic brain injuries functional imaging techniques may explain or predict postinjury neuropsychologic and cognitive deficits that are not explained by anatomic abnormalities detected by MRI or CT. ${ }^{[9]}$ SPECT study may thus allow us to understand the clinical correlations of the abnormalities detected in routine imaging findings like MRI and such correlations pertaining to RPL syndrome are reported in this communication.

\section{CASE REPORT}

A 31-year-old male presented with history of headache associated with recurrent episodes of vomiting for the past $24 \mathrm{~h}$. He developed 2 episodes of right focal motor to bilateral tonic-clonic seizures $3 \mathrm{~h}$ prior to admission. Following the seizures he remained in altered sensorium.

He had a prior medical history of medication-treated hypertension for the past 4 years. Renal biopsy was obtained 1 year prior to presentation and revealed focal segmental glomerular sclerosis with multifocal tubular atrophy. He was assessed by nephrologist for the same and was then placed on continuous ambulatory peritoneal dialysis along with antihypertensive therapy (amlodipine $5 \mathrm{mg} /$ day and prasozin $5 \mathrm{mg} /$ day). He was poorly compliant with his anti-hypertensive medicines and had discontinued them 1 week prior to this presentation.

On examination, his recorded vital parameters were: pulse: $142 / \mathrm{min}$, regular and blood pressure: 240/180 mmHg. General physical and cardiorespiratory examinations were unremarkable. Neurologically he was drowsy, arousable, but not obeying commands.
There was no word output. Optic fundi revealed no papilledema. There were features of grade 3 hypertensive retinopathy. There was right gaze palsy, right hemiplegia (grade 0/5 over the upper and lower limbs), bilaterally brisk deep tendon reflexes and extensor plantar responses.

Investigations revealed: blood urea $29 \mathrm{mg} / \mathrm{dL}$, serum creatinine $1.6 \mathrm{mg} / \mathrm{dL}$ and uric acid $8.8 \mathrm{mg} / \mathrm{dL}$. Urinalysis revealed albuminuria. Hemogram revealed leukocytosis (total leucocyte count 28,500 cells/cu.mm). Abdominal ultrasound (done 1 year ago) showed congenital pelviureteral junction obstruction in the right kidney with hydronephrotic sac, dilated renal pelvis and thinned renal parenchyma and the left kidney showed compensatory hypertrophy. MRI of the brain completed on day 2 of the illness revealed multiple lesions that were hypointense on T1-weighted images, hyperintense on T2-weighted and FLAIR images [Figure 1]. The lesions involved bilateral cerebellar hemispheres, brainstem, basal ganglia, posterior parieto occipital regions, corona radiata, centrum semiovale and splenium of corpus callosum with effacement of ventricular system and sulci. The lesions were moderately bright on diffusion weighted imaging (DWI with b value 600 ) and lower signal in ADC map. Anterior circulation was relatively spared. MR angiography revealed dolichoectasia with atheromatous dilatation of anterior and posterior circulation arteries without major vascular occlusion. Brain SPECT was performed on the 3rd day of illness after administration of $20 \mathrm{mCi}$ of Technetium-99m ethyl cysteinate dimer (99mTc-ECD). Images were acquired with a gamma camera, $1 \mathrm{~h}$ after tracer administration. Acquired images were then reconstructed in transaxial, saggital and coronal axes. Images revealed perfusion defects involving bilateral basal ganglia, left parietooccipital, right cerebellar and right occipital regions [Figure 2].

Intravenous sodium nitroprusside $(0.25 \mu \mathrm{g} / \mathrm{kg} / \mathrm{min}$ initial dose but then titrated up) was administered for hypertension. Intravenous fosphenytoin $(20 \mathrm{mg} / \mathrm{kg}$ of phenytoin equivalent) was given followed by maintenance phenytoin ( $5 \mathrm{mg} / \mathrm{kg} / \mathrm{day}$ ) therapy. There were no further recurrences of seizures. His blood pressure gradually decreased and oral antihypertensive therapy was instituted. His mental status improved by the second day after admission and limb power was grade $1 / 5$ on the 3rd day. The power of his motor movements rapidly improved subsequently and was grade $5 / 5$ on the 5 th day after admission and treatment. He remained asymptomatic since the 5th day after admission. At the time of discharge he was on a combination of prasozin $5 \mathrm{mg}$, clonidine $0.3 \mathrm{mg}$, labetolol $300 \mathrm{mg}$ and amlodepine $10 \mathrm{mg} /$ day for control of hypertension. 

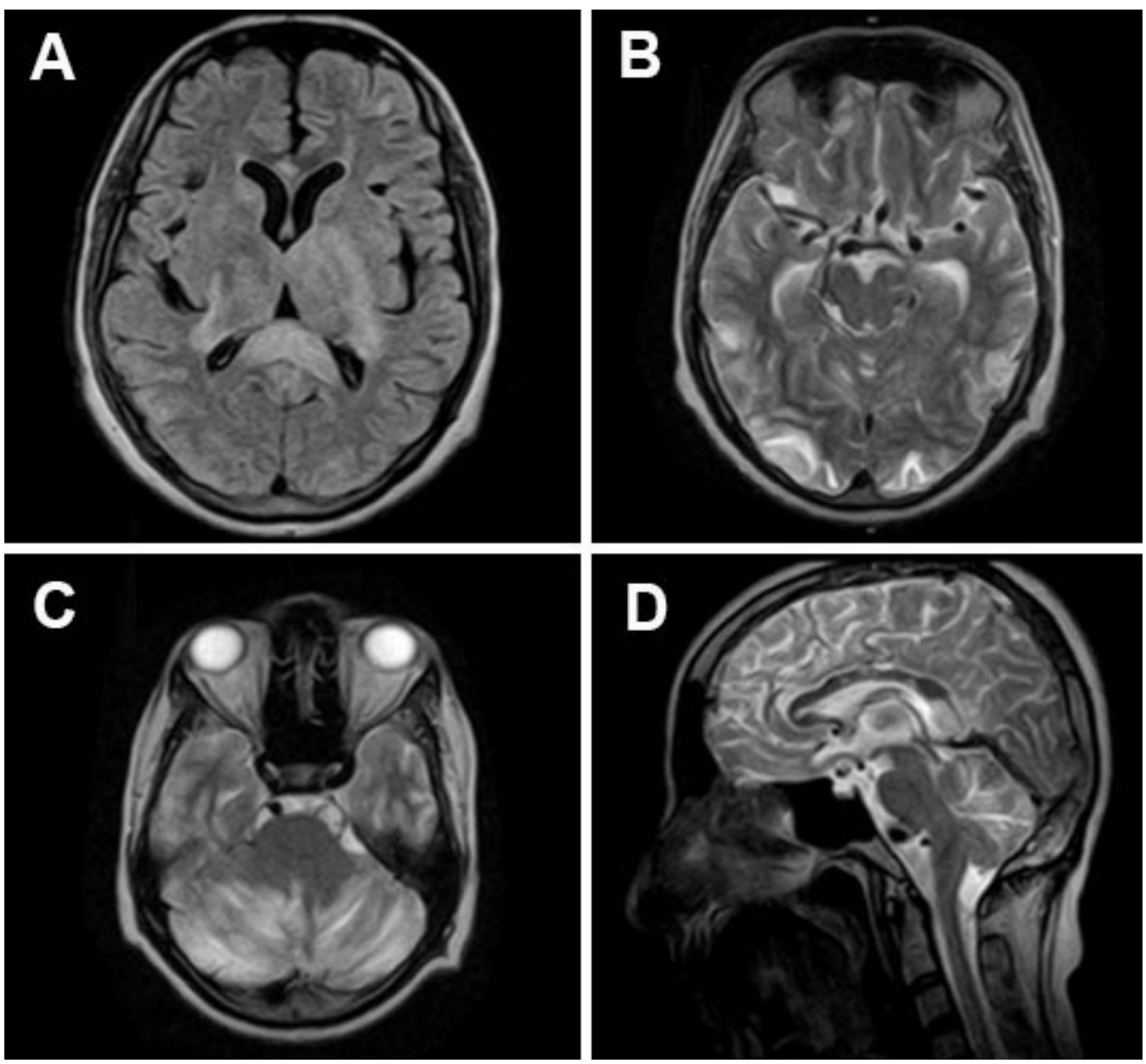

Figure 1: Magnetic resonance imaging in a patient with reversible posterior leukoencephalopathy. FLAIR axial (TR, $6160 \mathrm{~ms}$; TE, 30 ms; TI, 1100 ms; FOV $23 \mathrm{~cm} \times 23 \mathrm{~cm}$; matrix $256 \times 192$; slice thickness, $5 \mathrm{~mm}$ with $1 \mathrm{~mm}$ gap) (A); TSE T2-weighted (TR, 4500 ms; TE, 118 $\mathrm{ms}$; FOV $23 \mathrm{~cm} \times 23 \mathrm{~cm}$; matrix $256 \times 192$; slice thickness, $5 \mathrm{~mm}$ with $1 \mathrm{~mm}$ gap) axial (B and C) and sagittal images (D) are shown. Figure shows hyperintensities over both thalami, posterior limb of left internal capsule and corpus callosum on FLAIR axial image (A); hyperintense signals are seen over bilateral parieto-occipital cortices (B) and both cerebellar hemispheres (C) on T2 axial images; hyperintensities over corpus callosum and cerebellum are also seen in a T2 sagittal image (D) ${ }^{[16]}$

A repeat MRI done completed 2 weeks after the institution of treatment revealed resolution of the lesions over the cerebellar hemispheres, brainstem, basal ganglia and cortical regions. Follow up SPECT study could not be done.

\section{DISCUSSION}

This is a report of SPECT characteristics in a patient presenting with RPL syndrome. SPECT provides clinically useful information about brain perfusion and such data can especially be useful in conjunction with CT scan or MR imaging in diseases that cause flow abnormalities. The coupling between local metabolism and blood flow allows SPECT to provide indirect information about metabolism in focal areas of the brain. ${ }^{[10]}$ Hinchey et al. ${ }^{[1]}$ noted that while the radiological abnormalities were symmetric, the degree of involvement and the clinical manifestations were often asymmetric. Thus, in RPL syndrome where the defects are more functional than structural, SPECT abnormalities in the form of areas of hypoperfusion correlated well with the clinical abnormalities as was seen in this case. While MRI revealed bilateral parieto-occiptal and cerebellar hemispheric changes, SPECT images revealed predominant left hemispheric changes that correlated with the clinical picture of right hemiparesis and right gaze palsy and the bilateral basal ganglia lesions correlated with the bipyramidal signs.

Central to the pathogenesis of RPL syndrome is the failure of cerebral autoregulation and endothelial dysfunction. When the upper limit of autoregulation is exceeded arterioles dilate resulting in hyperperfusion that leads to breakdown of blood-brain barrier with focal transudation of fluid and petechial hemorrhages. ${ }^{[1]}$ Alternatively, in severe cases, it has been postulated that disordered cerebral autoregulation may lead to reactive focal vasoconstriction, thereby resulting in local hypoperfusion, cytotoxic edema, and cerebral infarction. ${ }^{[11]}$ Endothelial dysfunction has also been 


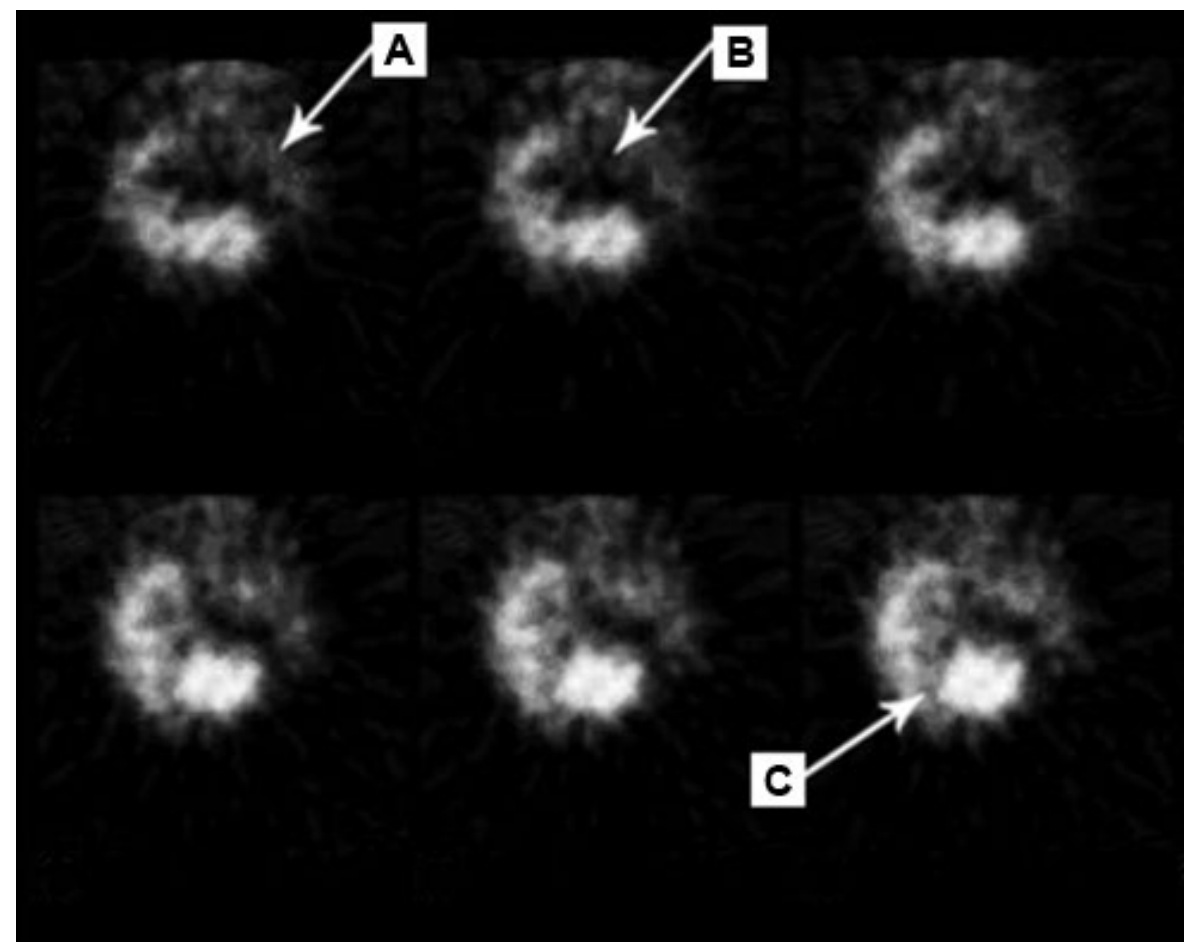

Figure 2: Brain single photon emission computerized tomography images obtained after administration of 20 mCi of $99 \mathrm{~m}$ Tc-ECD in a patient with reversible posterior leukoencephalopathy. Perfusion defects are seen involving the left fronto-parietal region (A), bilateral basal ganglia (B) and right cerebellum (C)

implicated in the pathophysiology of RPL syndrome, especially in cases associated with preeclampsia or cytotoxic therapies. ${ }^{[1]}$ Vasogenic edema related to this is the most common abnormality on neuroimaging in patients with RPL syndrome and involves the cortical and predominantly subcortical white matter in the posterior portions of the cerebral hemispheres, especially bilaterally in the parieto-occipital regions usually sparing the calcarine and paramedian occipitallobe structures. ${ }^{[1]}$ The latter feature helps to differentiate the syndrome from top-of-the basilar artery syndrome with involvement of posterior cerebral artery territories bilaterally. This differentiating feature was seen in our patient as well. In addition there was also involvement of bilateral cerebellar hemispheres, brainstem, basal ganglia, centrum semiovale, corona radiata, and splenium of corpus callosum. Involvement of the last two areas has not reported by earlier authors. However, clinical abnormalities correlating with such extensive radiological involvement were lacking in our patient.

Perfusion defects were seen over the right cerebellar region on SPECT imaging. Hypometabolism and hypoperfusion of the cerebellar cortex contralateral to the side of infarct usually occurs during the first two months after infarction and is referred to as crossed cerebellar diaschisis. Even relatively small lesions with mild metabolic depression also have been noted to produce contralateral cerebellar hypometabolism.
It is thought to be due to interruption of corticoponto-cerebellar connections due to the infarct which then causes deafferentation and transneural metabolic depression of the contralateral cerebellar hemisphere. ${ }^{[12]}$ It could be proposed that the right cerebellar perfusion defects seen on SPECT were an early manifestation of this phenomenon although the interval between the onset of the disease and the time of SPECT is short in our patient. While MRI revealed bilateral cerebellar lesions, only SPECT revealed abnormalities corresponding to the left cerebral hemispheric involvement with corresponding right cerebellar hemispheric changes. Thus, SPECT is a useful tool in understanding the pathophysiological abnormalities underlying the disease. It has been known that post-traumatic psychosis is associated with perfusion defects in frontal lobes even when the routine imaging studies like MRI and CT scan are normal. ${ }^{[9]}$ Thus SPECT better localizes areas of clinical injury and may prove to be a useful tool in understanding the pathophysiological abnormalities underlying RPL syndrome.

The advantage of SPECT is its ability to detect ischemic tissue before irreversible damage occurs. ${ }^{[13]}$ For instance, it has been shown that SPECT is a sensitive indicator of perfusion and is considered superior to anatomic imaging modalities such as CT or MRI in detecting acute ischemic stroke in the first few hours following the events. Immediately after 
acute stroke, a focal or regional area of hypoperfusion or no perfusion will be seen which is larger than the lesion that is later seen on CT or MRI. Brain perfusion SPECT patterns may predict the outcome of stroke patients and thus help in the selection of candidates for fibrinolytic therapy as well as decompressive hemicraniectomy. ${ }^{[14,15]}$ Functional imaging studies like perfusion and diffusion weighted MR imaging and MR spectroscopy could also be utilized to detect ischemic changes. ${ }^{[16-19]}$ Hyperintense signals are seen in areas of both vasogenic edema and infarction on routine T2 weighted images. However, on diffusion weighted imaging, vasogenic areas demonstrate increased diffusion and cyotoxic edema seen in infarcted areas demonstrate decreased diffusion. In our patient repeat MR imaging revealed resolution of the lesions suggesting reversible ischemia rather than established infarct. Thus it is understandable that functional studies like SPECT may enhance the understanding of the extent and nature of the lesions typical of the syndrome. Follow up SPECT study however could not be done in the patient.

In conclusion, SPECT findings in our patient with RPL syndrome correlated positively with the presented clinical deficits: advantage of SPECT study over routine MRI is its good correlation with clinical abnormalities. MRI abnormalities, in contrast to SPECT findings, are extensive and poorly correlate with clinical deficits. Thus SPECT may be an invaluable tool to understand the morphological and functional changes in RPL syndrome.

\section{Financial support and sponsorship None.}

\section{Conflicts of interest}

There are no conflicts of interest.

\section{Patient consent \\ Patient consent was obtained from the patient.}

\section{Ethics approval}

Data collection in our study involving the patient is consistent with the ethical standards of the institution's ethics committee.

\section{REFERENCES}

1. Hinchey J, Chaves C, Appignani B, Breen J, Pao L, Wang A, Pessin MS, Lamy C, Mas JL, Caplan LR. A reversible posterior leukoencephalopathy syndrome. N Engl J Med 1996;334:494-500.
2. Casey SO, Sampaio RC, Michel E, Truwit CL. Posterior reversible encephalopathy syndrome: utility of fluid-attenuated inversion recovery MR imaging in the detection of cortical and subcortical lesions. AJNR Am J Neuroradiol 2000;21:1199-206.

3. Tajima Y, Isonishi K, Kashiwaba T, Tashiro K. Two similar cases of encephalopathy, possibly a reversible posterior leukoencephalopathy syndrome: serial findings of magnetic resonance imaging, SPECT and angiography. Intern Med 1999;38:54-8.

4. Nishigaki Y, Inoue M, Jimi T, Wakayama Y, Yoshiba M. Reversible posterior leukoencephalopathy in a patient receiving cyclosporin therapy. Rinsho Shinkeigaku 1999;39:360-3. (in Japanese)

5. Naidu K, Moodley J, Corr P, Hoffmann M. Single photon emission and cerebral computerised tomographic scan and transcranial Doppler sonographic findings in eclampsia. Br J Obstet Gynaecol 1997;104:1165-72.

6. Schwartz RB, Jones KM, Kalina P, Bajakian RL, Mantello MT, Garada B, Holman BL. Hypertensive encephalopathy: findings on CT, MR imaging, and SPECT imaging in 14 cases. AJR Am J Roentgenol 1992;159:379-83.

7. Strandgaard S, Paulson OB. Cerebral autoregulation. Stroke 1984;15:413-6.

8. Dougall NJ, Bruggink S, Ebmeier KP. Systematic review of the diagnostic accuracy of 99mTc-HMPAO-SPECT in dementia. Am J Geriatr Psychiatry 2004;12:554-70.

9. Raji CA, Tarzwell R, Pavel D, Schneider H, Uszler M, Thornton J, van Lierop M, Cohen P, Amen DG, Henderson T. Clinical utility of SPECT neuroimaging in the diagnosis and treatment of traumatic brain injury: a systematic review. PLoS One 2014;9:e91088.

10. Prichard JW, Brass LM. New anatomical and functional imaging methods. Ann Neurol 1992;32:395-400.

11. Koch S, Rabinstein A, Falcone S, Forteza A. Diffusion-weighted imaging shows cytotoxic and vasogenic edema in eclampsia. $A J N R$ Am J Neuroradiol 2001;22:1068-70.

12. Reivich M. Crossed cerebellar diaschisis. AJNR Am J Neuroradiol 1992;13:62-4.

13. Alavi A, Hirsch LJ. Studies of central nervous system disorders with single photon emission computer tomography and positron emission tomography: evolution over the past 2 decades. Semin Nucl Med 1991;21:58-81.

14. Bhattarai S, Ning ZX, Tuerxun T. EEG and SPECT Changes in acute ischemic stroke. J Neurol Neurophysiol 2014;5:190.

15. Masdeu JC, Arbizu J. Brain single photon emission computed tomography: technological aspects and clinical applications. Semin Neurol 2008;28:423-34.

16. Brubaker LM, Smith JK, Lee YZ, Lin W, Castillo M. Hemodynamic and permeability changes in posterior reversible encephalopathy syndrome measured by dynamic susceptibility perfusion-weighted MR imaging. AJNR Am J Neuroradiol 2005;26:825-30.

17. Schaefer PW, Buonanno FS, Gonzalez RG, Schwamm LH. DiffusionWeighted Imaging discriminates between cytotoxic and vasogenic edema in a patient with eclampsia. Stroke 1997;28:1082-5.

18. Russell MT, Nassif AS, Cacayorin ED, Awwad E, Perman W, Dunphy F. Gemcitabine-associated posterior reversible encephalopathy syndrome: MR imaging and MR spectroscopy findings. Magn Reson Imaging 2001;19:129-32.

19. Covarrubias DJ, Luetmer PH, Campeau NG. Posterior reversible encephalopathy syndrome: prognostic utility of quantitative diffusionweighted MR images. AJNR Am J Neuroradiol 2002;23:1038-48. 